https://doi.org/10.15407/ujpe63.5.396

V.V. MYKHAYLOVSKYY, V.I. SUGAKOV

Institute for Nuclear Research, Nat. Acad. of Sci. of Ukraine

(47, Prosp. Nauky, Kyiv 03680,Ukraine; e-mail: mykhay@kinr.kiev.ua)

\title{
PULSES OF THE EXCITONIC CONDENSED PHASE IN SEMICONDUCTORS WITH DOUBLE QUANTUM WELL AT STEADY PUMPING: SIZE EFFECTS ${ }^{1}$
}

\begin{abstract}
The conditions, under which the generation and movement of solitons (regions of exciton condensed phases) occurs in double quantum wells of semiconductors under a stationary pumping and in the presence of an external driving force, are analyzed. It is shown that there is a minimal size of the system, in which a state with moving solitons can be created. The dependence of the minimum value of the driving force necessary for the generation of moving solitons on the size of the system is found.

Ke ywords: luminescence, indirect excitons, double quantum well, self-organization, traveling pulses.
\end{abstract}

\section{Introduction}

The formation of spatial structures in the exciton density distribution in double quantum wells is an intriguing phenomena in the physics of strong exciting systems. Firstly, unusual effects were observed in works [1, 2]. In the work by Butov et al. [1], the periodic distribution emission was revealed from a ring situated around a laser spot in the double quantum well on the base of AlGaAs. In the work by Timofeev et al. [2], the periodic distribution emission was shown along the perimeter of a window, through which the quantum well was excited. It seems that the indirect excitons in double quantum wells are a perspective object for the observation of the exciton Bose-Einstein condensation: they have the integer spin and the same orientation of dipole moments, which leads to the repulsion interaction between excitions, manifesting itself in a short-wave shift of the luminescence with increasing the exciton pumping. But the attempts to explain these phenomena and the appearance of the structures in the other special created non-homogeneous systems from the viewpoint of the Bose-Einstein condensation were not successful so far. There are investigations [3-12], in which different mechanisms of the appearance of periodic structures in multiexciton systems are suggested. However, they have particular nature and do not touch on a variety of the observed effects.

(c) V.V. MYKHAYLOVSKYY, V.I. SUGAKOV, 2018
In works $[13,14]$, the model were suggested, according to which the appearance of the condensed phase of indirect excitons is connected with the attractive interaction between excitons. The dipole-dipole repulsion causes a shortwave shift of the excitonic luminescence frequency with increasing the exciton densirty. But, at some exciton density, the exchange and van der Waals interactions prevail over the dipoledipole repulsion, and the excitonic condensed phase may arise. Such conclusion is confirmed by 1) the presence of binding states of two indirect excitons obtained by quantum mechanical calculations [1517] and 2) calculation of many-particle problem of the system of indirect excitons at zero temperature, taking the correlation and exchange interactions into account [18]. But the considered system is nonequilibrium due to the presence of a pumping and the finite value of the exciton lifetime. In works $[13$, 14, 19], a generalization of the phase transition theory was presented for non-equilibrium systems, and the explanation of the results of experiments in $[1$, $2]$ was given. In addition, the analysis of another experiments was fulfilled. The peculiarities of the appearance of a structure in the luminescence of indirect excitons in the presence of a periodic potential [20] were presented in [21]. The structures that

1 The paper was presented at the XXIII Galyna Puchkovska International School-Seminar "Spectroscopy of Molecules and Crystals".

ISSN 2071-0194. Ukr. J. Phys. 2018. Vol. 63, No. 5 
arise in the luminescence from double quantum wells with electrodes of the different form [22, 23] were explained in [24]. The explanation of the fragmentation of the inner ring in a laser sport observed in [25] was given in [26]. The developed theory allows one to predict the appearance of solitons [27], which present the localized regions of the exciton condensed phase, the movement of solitons in external fields [28, 29 , the generation of autowaves in the exciton condensed phases (solitons) at a steady state irradiation of double quantum wells, and the movement of the autowaves on macroscopic distances [30]. According to [27], several conditions should be realized for the appearance of the generation. The system should consist of 1) long regions irradiated by the pumping on the level of bistability, at which the two types of the exciton distribution may exist - uniform and non-uniform, 2) small region irradiated with a higher pumping, the generation arises in this region; 3 ) the presence of a driving force, which turns off the excitons from one region to another, where they move in the form of the exciton condensed phase pulses. The flux of pulses may be controlled by the switching-on or switching-off the pumping.

In the presented work, the peculiarities of the pulse generation process and the values of parameters, at which it occurs, are studied. Particularly, the thresholds of appearance and their dependence on the sizes of irradiated regions and on the properties of the boundaries are investigated.

\section{Analysis of the Stability: Dependence on the Lifetime and the Density of Excitons}

We now consider the inhomogeneous nonstationary distribution of excitons in the presence of a pumping with regard for the finite lifetime of particles. Usually, the many-particle exciton problem is described by the Gross-Pitaevskii equation. However, in a given nonequilibrium nonuniform system, the chemical potential is inhomogeneous. Even under a uniform excitation, the structures with different exciton densities arise, and there is an influx of excitons from one region to another. The current occurs due to the scattering of particles by phonons and impurities. The process is incoherent, and the resulting structures are dissipative. Therefore, to describe the dynamics of excitons, we use, like the previous papers, the equation involving the conservation of the number of excitons, interaction between excitons, pumping, and finite life- time of excitons:

$\frac{\partial n}{\partial t}+\operatorname{div} \mathbf{j}=G-\frac{n}{\tau_{\mathrm{ex}}}$

Here, $n$ is the exciton concentration, $\mathbf{j}$ is the exciton current, $G$ is the number of excitons produced per unit of time and unit of surface (pumping), and $\tau_{\text {ex }}$ is the exciton lifetime. We suggest that the state of local equilibrium is realized, and the system can be described by the free energy, which depends on the exciton density. The current of excitons can be found [31] in terms of the gradient of the chemical potential $\mu$, linking the exciton mobility with the exciton diffusion coefficient $D$ by the Einstein relation

$\mathbf{j}=-\frac{n D}{k_{\mathrm{B}} T} \nabla \mu$.

The chemical potential $\mu$ is found from the free energy $F$ as

$\mu=\frac{\delta F}{\delta n}$.

The free energy is approximated by the expression

$F=\int d S\left[\frac{K}{2}(\nabla n)^{2}+f(n)+n V(x)+\right.$

$\left.+k_{\mathrm{B}} T n(\ln n-1)\right]$,

where $\frac{K}{2}(\nabla n)^{2}$ is the energy of heterogeneity, $n V(x)$ is the energy of interaction of excitons with an external field with potential $V(x)$, and $f(n)$ is the free energy density. We will approximate it as a series of $n$. In our works, we used expansions up to $n^{4}$ or up to $n^{6}$ in the description of exciton systems. Both representations correctly describe the appearance and properties of the radiation from the spatial structures of excitons. However, the absence of quantitative experimental values of the distribution of exciton densities does not allow one to give preference to one or other presentation of $f(n)$. In this paper, we approximate it in the form

$f(n)=\frac{a}{2} n^{2}+\frac{b}{4} n^{4}+\frac{c}{6} n^{6}$.

The parameters $a, b$, and $c$ in Eq. (4) are chosen so that the free energy as a function of the exciton density has a minimum corresponding to the condensed phase and describes both the existence of the phase transition and the spectral shift to higher frequencies with increasing $n$ due to the dipole-dipole repulsion. To accomplish this, the following conditions 


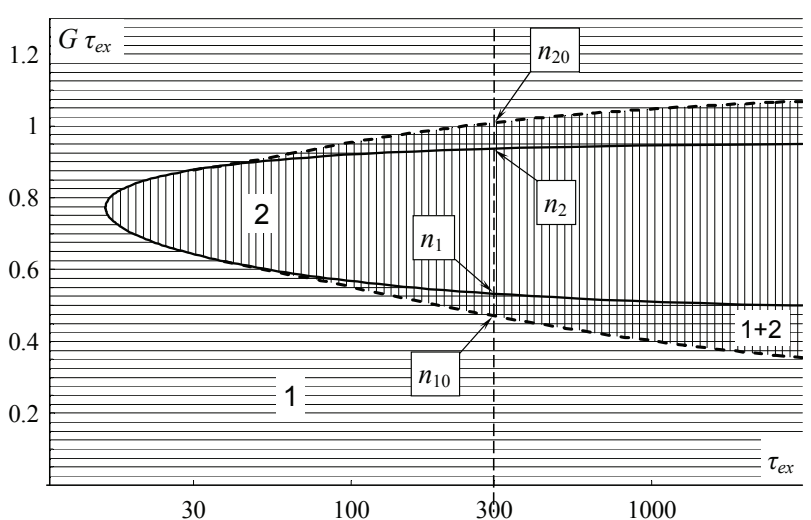

Fig. 1. Diagram of the stability of uniform and nonuniform exciton distributions on the plane of the lifetime of the excitons and the pump intensity. The following parameters were used: $b=-1.9, D=0.03$ (dimensionless values, see the text for details). Area with stable uniform distribution is filled by horizontal lines. Area with stable periodic distribution is filled by vertical lines. Area with both stable distributions allows the existence of a soliton solution

should be valid: $a>0, b<0, c>0$. Note that, at $T=0$ and for a uniform system, the free energy (4) has form obtained in [18] by quantum mechanical calculations.

The last term in (3) is the free energy of an ideal gas. After its substitution into the equation for the concentration of excitons, it gives the usual linear term for the diffusion. In the region of large values of $n$, this term is unimportant; it is introduced to describe the density distribution in a region with small values (far from the irradiation region).

The term with $V(x)$ specifies the inhomogeneity introduced by external conditions ( $x$ is the coordinate along the quantum well surface); for example, such an inhomogeneity can be created by the inhomogeneity of an external electric field, which separates an electron and a hole in different wells and affects the energy of an exciton, or by applying a pressure.

To make the analysis simpler, the dimensionless variables are introduced. Let us define

$l_{0}=\sqrt{\frac{K}{a}}, \quad n_{0}=\left(\frac{a}{c}\right)^{1 / 4}, \quad t_{0}=\frac{K_{\mathrm{B}} T K}{D n_{0} a^{2}}$.

We use

$\tilde{x}=\frac{x}{l_{0}}, \quad \tilde{t}=\frac{t}{t_{0}}, \quad \tilde{n}=\frac{n}{n_{0}}, \quad \tilde{G}=G \frac{t_{0}}{n_{0}}, \quad \tilde{\tau}_{\mathrm{ex}}=\frac{\tau_{\mathrm{ex}}}{t_{0}}$, $\tilde{D}=D \frac{t_{0}}{l_{0}^{2}}, \quad \tilde{b}=\frac{b}{\sqrt{a c}}, \quad \tilde{V}=\frac{V}{a n_{0}}$.
The $\sim$ sign will be omitted in what follows. In the dimensionless units, the equation for the density of excitons (1) takes the form

$$
\begin{aligned}
& \frac{\partial n}{\partial t}=D \Delta n+G-\frac{n}{\tau_{\mathrm{ex}}}+\left(n+3 b n^{3}+5 n^{5}\right) \Delta n+ \\
& +\left(1+9 b n^{2}+25 n^{4}\right)(\nabla n)^{2}-n \Delta \Delta n- \\
& -\nabla n \nabla \Delta n-F \nabla n,
\end{aligned}
$$

where $F=-\frac{\partial V(x)}{\partial x}$ is called the driving force.

Possible solutions of Eq. (6) are analyzed in [27] in the absence of a driving force. We need a characterization of these solutions to analyze the effect of the driving force and the parameters of the irradiated regions on the process of generation of condensed phases. We consider one-dimensional solutions of Eq. (6) in a quantum well, where a region $L$ in length is irradiated. One-dimensional solutions can be realized, for example, by irradiating a quantum well through a gap in the electrode that creates an electric field in the well. We consider a system, in which the pump region is restricted, with no pumping outside. The concentration of excitons is still described by Eq. (6), which leads to the exponential decay of the exciton concentration to zero, and the zero boundary conditions are fulfilled at large distances from the irradiated region.

A numerical analysis of the solutions of Eq. (6) leads to a diagram on the plane of the lifetime of the excitons and the pump intensity presented in Fig. 1. The parameter $b=-1.9$ in Fig. 1 is taken such that the condensed phase exists in the system, i.e., slightly above the threshold. The estimate of the dimensionless diffusion coefficient $\tilde{D}=0.03$ is taken from (5) for the typical values of the dimensional parameters $t_{0}=10^{-10} \mathrm{~s}, l_{0}=2 \times 10^{-8} \mathrm{~cm}$, $D=10 \mathrm{~cm}^{2} / \mathrm{s}$. Let us analyze the dynamics of the change in states with increasing the pumping for particles with a definite lifetime $\tau_{\mathrm{ex}}$. The typical lifetime is about $10^{-8}-10^{-7} \mathrm{~s}$; so, according to (5), $\tau_{\mathrm{ex}}$ is about $100-1000$. Let $\tau_{\mathrm{ex}}=300$, then a change in the density with increasing the pumping is determined by the vertical dashed curve shown in Fig. 1. There are several important values on this way. At small values of the density $G \tau_{\text {ex }}<n_{1}$, the gas phase of excitons is realized in the system, which means a homogeneous exciton distribution. In the region $n_{1}<G \tau_{\mathrm{ex}}<n_{2}$, the homogeneous distribution is unstable, and a periodic distribution of the exciton density arises in the 


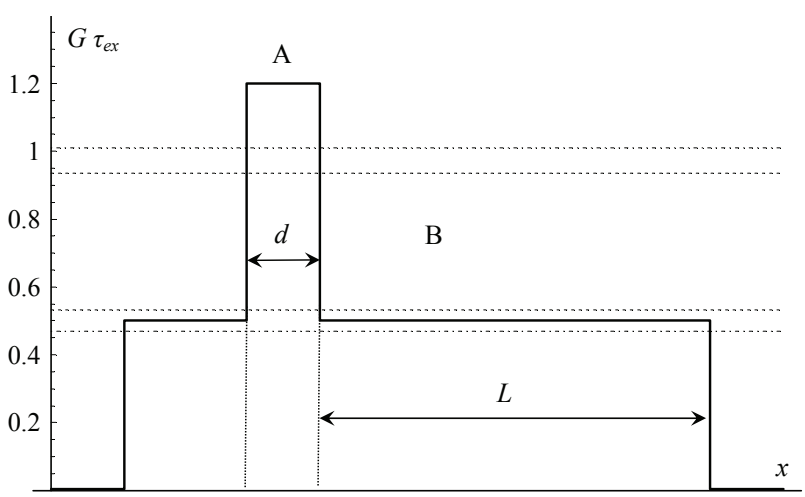

Fig. 2. Scheme of the nonuniform irradiation as a function of the pumping versus the coordinate. Region A is illuminated intensively to the level of the condensed phase $\left(G \tau_{\mathrm{ex}}=1.2\right)$. Region B is illuminated to the level of the bistability $\left(G \tau_{\mathrm{ex}}=\right.$ $0.5)$. Dashed horizontal lines represent the values of $n_{10}, n_{1}$, $n_{2}$, and $n_{20}$

system. The period increases with the pumping. For $G \tau_{\text {ex }}>n_{2}$, the condensed phase of excitons is formed in the system, which appears to be homogeneous again. Calculations show that there are bistability areas near the borders of instability $\left(n_{10}<G \tau_{\text {ex }}<n_{1}\right.$ and $n_{2}<G \tau_{\text {ex }}<n_{20}$ ), where both homogeneous and periodic distributions of excitons are stable. In these regions, the exciton distributions can also be realized in the form of single peaks (solutions in this form were obtained in [27]). They are called static solitons [32].

In the linear external potential $(F=$ const $\neq 0)$, the considered structures move, by forming autowaves. In the case where the boundary effects can be neglected, the replacement $n(x, t)=n_{\text {st }}(x-F t)$ in Eq. (6) allows us to express the solution $n(x, t)$ for $F \neq 0$ in terms of the stationary solution $n_{\text {st }}(x)$ for $F=0$. The solution has the form of a wave moving with velocity equal to $F$.

\section{Simulation Results}

Following [30], we consider a semiconductor with a double quantum well, in which the creation of excitons occurs in several adjacent regions (Fig. 2). A narrow region $d$ in width is denoted as $\mathrm{A}$; the second wide region (B) $L$ in width is irradiated with an intensity creating the exciton density with its value in the bistability region (i.e., $n_{10}<G \tau_{\text {ex }}<n_{1}$, see Fig. 1). This region can be in a state of uniform exiton distribution or in the exciton soliton state. We
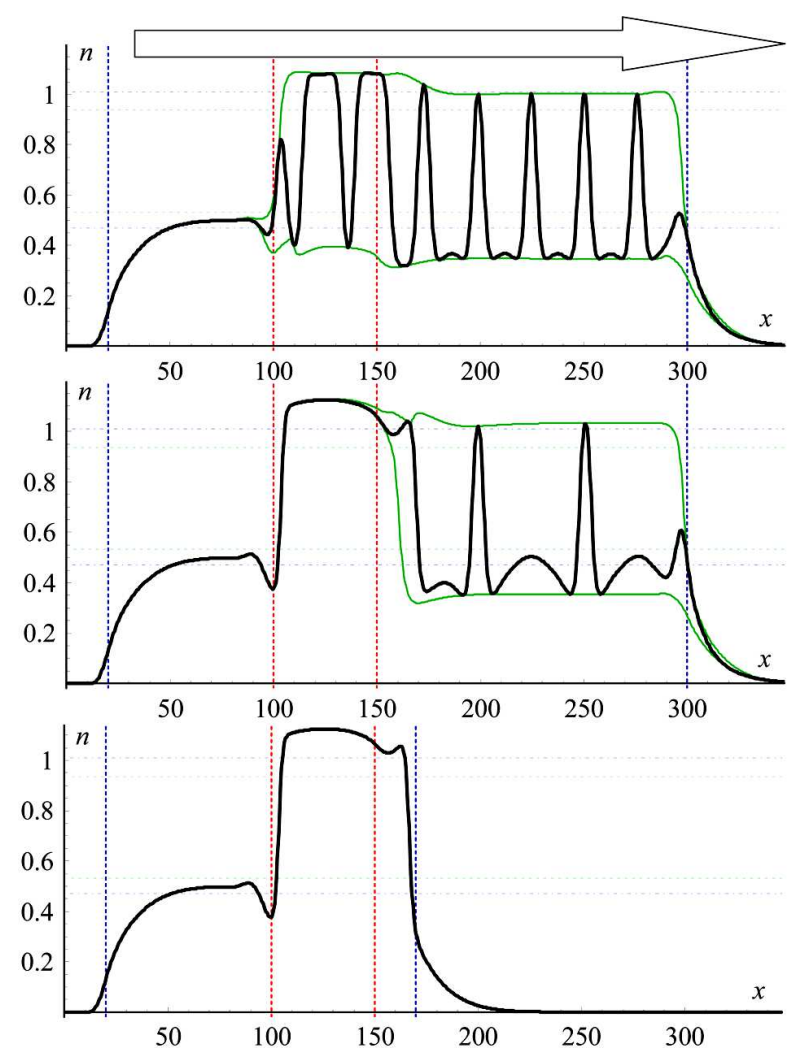

Fig. 3. Spatial distribution of the density of excitons in a steady state with the driving force $F=0.028$. In time, the peaks - islands of a denser phase - move to the right with the velocity equal to $F$. Thin green lines show the envelopes of propagating peaks. Vertical red lines limit the region with strong pumping of excitons, vertical blue lines - a region with the intermediate pumping of excitons. On the top graph, the pumping in region $\mathrm{A}$ corresponds to the instability $\left(G \tau_{\mathrm{ex}}=\right.$ $=0.9$ ). On the lower graph, which corresponds to a small size of region $\mathrm{B}$, the concentration of excitons appears to be stationary

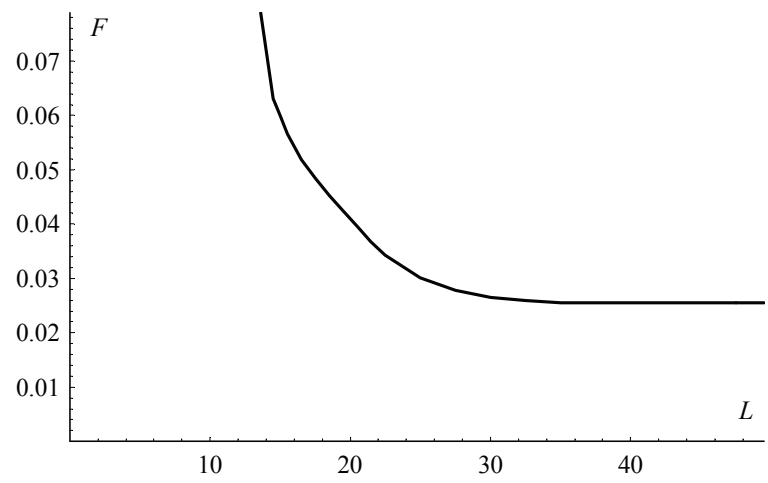

Fig. 4. Minimum value of the driving force necessary for the generation of autowaves as a function of the size of region $\mathrm{B}$ 
will see that the state in region $B$ is regulated by the irradiation intensity of region $\mathrm{A}$. If the pumping is absent, the exciton concentration $n$ exponentially decays to zero due to the finite lifetime of excitons.

Let us study the dependence of the process of soliton generation in region $\mathrm{B}$ on the sizes of the region and the magnitude of an applied driving force. In the absence of the irradiation in region $\mathrm{A}$, there are no solitons in region $\mathrm{B}$, and the luminescence from this region is homogeneous. Let us increase the irradiation intensity in region $\mathrm{A}$. When the intensity appears to correspond to the instability region, the exciton density pulses appear in region $\mathrm{A}$, travel through region $\mathrm{B}$, and disappear at the boundary of the irradiation region (Fig. 3, top). When the pumping intensity in region A corresponds to the excitation of the condensed phase, the situation is similar, when the size of region $\mathrm{B}$ is large, and the value of the driving force is above some threshold [30]. In this case, the pulses of high-density excitons appear at the boundary between regions $\mathrm{A}$ and $\mathrm{B}$ (Fig. 3, middle). If we lower the irradiation intensity in region $\mathrm{A}$, then no new pulses appear. When all pulses go away from region $\mathrm{B}$, the exciton density returns to the uniform distribution.

If the size of region B is small, larger driving forces are needed to start the generation from the region pumped to the condensed phase. There is a minimum value of the size of the region, at which the generation is possible. Let the size of region $\mathrm{B}$ be less than the critical value. When the irradiation of region $\mathrm{A}$ is switched-on, a certain stationary distribution of the exciton density is established in the system (Fig. 3, bottom). However, if the parameters of the system are such that the generation is started, it takes place with the same frequency and shape as in the absence of a boundary.

Figure 4 shows the critical values of the driving force that causes the generation of autowaves as a function of the size of region B. Smaller regions B require larger driving forces to trigger the generation. If the region of a pumping is too small, the autowave generation does not occur even for large values of the driving force.

Let us make estimations of possible soliton velocities in the system. In dimensional units, the velocity is equal to $v=-D F /(k T)$. In the case of $D=100 \mathrm{~cm}^{2} / \mathrm{s}, T=2 \mathrm{~K}, F=10^{-3} \mathrm{eV} /(100 \mu \mathrm{m})$, we obtain $v=5.7 \times 10^{4} \mathrm{~cm} / \mathrm{s}$.

\section{Conclusions}

The conditions of generation and motion of exciton solitons in the double quantum wells of semiconductors under a constant pumping and the presence of an external driving force have been analyzed. Solitons are localized regions (islands) of the condensed phase of excitons that appears under the certain conditions of pumping. The generation of solitons by a stationary pumping was proposed in [30] and is based on the fact that, in a definite pumping region, the system is in a bistable state - one solution is uniform, another solution is with solitions, which move in the presence of an inhomogeneous potential (driving force). With the help of an additional pumping, which irradiates the neighboring region, it is possible to transfer the system from one state to another one - from a state without solitons to a state with moving solitons and vice versa. In this paper, we have investigated the generation of solitons, depending on the size of the system. It is shown that there exists a minimal size of the system, for which a state with moving solitons can be created. The dependence of the minimum value of the driving force necessary for the generation of solitons on the size of the regions is found.

1. L.V. Butov, A.C. Gossard, D.S. Chemla. Macroscopically ordered state in an exciton system. Nature 418, 751 (2002).

2. A.V. Gorbunov, V.B. Timofeev. Collective state in a Bose gas of interacting interwell excitons. JETP Lett. 83, 146 (2006).

3. L.S. Levitov, B.D. Smons, L.V. Butov. Pattern formation as a signature of quantum degeneracy in a cold exciton system. Phys. Rev. Lett. 94, 176404 (2005).

4. A.V. Paraskevov, T.V. Khabarova. On the microscopic theory of the exciton ring fragmentation. Phys. Lett. A $\mathbf{3 6 8}$, 151 (2007).

5. R.B. Saptsov. On the instability of a homogeneous state of a weakly interacting Bose gas under external cooling. JETP Lett. 86, 687 (2008).

6. C.S. Liu, H.G. Luo, W.C. Wu. Pattern formation of indirect excitons in coupled quantum wells. J. Phys. Condens. Matter 18, 9659 (2006).

7. C.S. Liu, H.G. Luo, W.C. Wu. Theoretical modeling of spatial- and temperature-dependent exciton energy in coupled quantum wells. Phys. Rev. B 80, 125317 (2010).

8. V.K. Mukhomorov. On the possibility of realizing a periodic low-density spatial distribution of excitons. Phys. Solid State 52, 241 (2010).

9. J. Wilkes, E.A. Muljarov, A.L. Ivanov. Drift-diffusion model of the fragmentation of the external ring structure in the photoluminescence pattern emitted by indirect ex-

ISSN 2071-0194. Ukr. J. Phys. 2018. Vol. 63, No. 5 
citons in coupled quantum wells. Phys. Rev. Lett. 109 , 187402 (2012).

10. S.V. Andreev. Thermodynamic model of the macroscopically ordered exciton state. Phys. Rev. Lett. 110, 146401 (2013).

11. V.S. Babichenko, I.Ya. Polishchuk. Coulomb correlations and electron-hole liquid in double quantum wells. JETP Lett. 97, 726 (2013) .

12. V.S. Babichenko, I.Ya. Polishchuk. Quantum phase transition of electron-hole liquid in coupled quantum wells. Phys. Rev. B 94, 165304 (2016).

13. V.I. Sugakov. Islands of exciton condensed phases in a twodimensional system, the distribution of their sizes and coherence in position. Solid State Commun. 134, 63, (2005).

14. V.I. Sugakov. Exciton condensation in quantum wells: Temperature effects. Phys. Solid State 48, 1984 (2006).

15. M.Y. J. Tan, N.D. Drummond, R.J. Needs. Exciton and biexciton energies in bilayer systems. Phys. Rev. B 71, 033303 (2005).

16. Ch. Shindler, R. Zimmermann. Analysis of the excitonexciton interaction in semiconductor quantum wells. Phys. Rev. B 78, 045313 (2008).

17. A.D. Meyertholen, M.M. Fogler. Biexcitons in twodimensional systems with spatially separated electrons and holes. Phys. Rev. B 78, 235307 (2008).

18. Yu.E. Lozovik, O.I. Berman. Phase transitions in a system of two coupled quantum wells. JETP Lett. 64, 573 (1996).

19. A. A. Chernyuk, V. I. Sugakov. Ordered dissipative structures in exciton systems in semiconductor quantum wells. Phys. Rev. B 74, 085303 (2006).

20. M. Remeika, J.C. Graves, A.T. Hammack, A.D. Meyertolen, M.M. Fogler, L.V. Butov, M. Hanson, A.C. Gossard. Localization-delocalization transition of indirect excitons in lateral electrostatic lattices. Phys. Rev. Lett. 102, 186803 (2009)

21. A.A. Chernyuk, V.I. Sugakov. Exciton phase transitions in semiconductor quantum wells with disc-shaped electrode. Solid State Commun. 149, 2185 (2009).

22. V.B. Timofeev, A.V. Gorbunov, D.A. Demin. BoseEinstein condensation of dipolar excitons in lateral traps. Low Temp. Phys. 37, 179 (2011).

23. A.V. Gorbunov, V.B. Timofeev. Phase diagram of the Bose condensation of dipolar excitons in GaAs/AlGaAs quantum-well heterostructures. JETP Lett. 96, 143 (2012).
24. V.V. Tomylko, I.Yu. Goliney, A.A. Chernyuk, V.I. Sugakov. Exciton density pattern formation in laser irradiated quantum wells under electrodes of various shapes. Low Temp. Phys. 40, 975 (2014).

25. M. Remeika, A.T. Hammack, S.V. Poltavtsev, L.V. Butov et al. Pattern formation in the exciton inner ring. Phys. Rev. B 88, 125307 (2013).

26. A.A. Chernyuk, V.I. Sugakov, V.V. Tomylko. Model of fragmentation of the exciton inner ring in semiconductor quantum wells. Phys. Rev. B 90, 205308 (2014).

27. V.I. Sugakov. Exciton condensation in quantum wells. Selforganization against Bose-condensation. Ukr. J. Phys. 56, 1124 (2011)

28. V.I. Sugakov. Ordered structures of exciton condensed phases in the presence of an inhomogeneous potential. J. Phys. Condens. Matter 21, 275803 (2009).

29. O.I. Dmytruk, V.I. Sugakov. Amplification and passing through the barrier of the exciton condensed phase pulse in double quantum wells. Physica B 436, 80 (2014).

30. V. Mykhaylovskyy, V. Sugakov, I. Goliney. Excitation of pulses of excitonic condensed phase at steady pumping. J. Nanophotonics 10, 033504 (2016).

31. G. Nicolis, I. Prigogine. Self-Organization in Non-Equilibrium Systems (Wiley, 1977).

32. B.S. Kerner, V.V. Osipov. Autosolitons. Sov. Phys. Usp. 32, 101 (1989).

Received 27.02.18

\section{В.В. Михайловсъкий, В.Й. Сугаков}

ІМПУЛЬСИ ЕКСИТОННОї КОНДЕНСОВАНОЇ ФАЗИ В НАПІВПРОВІДНИКАХ З ПОДВІЙНИМИ КВАНТОВИМИ ЯМАМИ ПРИ СТАЦІОНАРНОМУ НАКАЧУВАННI: РОЗМІРНІ ЕФЕКТИ

$\mathrm{P}$ е $з$ ю м е

Проаналізовано умови, за яких виникає генерація і рух солітонів (областей екситонних конденсованих фаз) в подвійних квантових ямах напівпровідників при стаціонарній накачці і наявності зовнішньої тянучої сили. Показано, що існує мінімальний розмір системи, при якому стан з рухомими солітонами може бути створений. Знайдена залежність мінімального значення тянучої сили, необхідної для генерації рухомих солітонів, від розмірів системи. 